EPiC Series in Engineering
Volume 3, 2018, Pages 131-138
HIC 2018. 13th International
Conference on Hydroinformatics

\title{
Reformulation of 2D DG2 scheme for shallow water modelling
}

\author{
Janice Lynn Ayog ${ }^{1,2^{*}}$ and Georges Kesserwani ${ }^{1}$ \\ ${ }^{1}$ Civil \& Structural Engineering, University of Sheffield, Sheffield S1 3JD, United Kingdom \\ ${ }^{2}$ Faculty of Engineering, Universiti Malaysia Sabah, Jln UMS, 88400 Kota Kinabalu, Sabah, \\ Malaysia \\ jlayog1@sheffield.ac.uk, g.kesserwani@sheffield.ac.uk
}

\begin{abstract}
This paper presents a reformulation of the 2D second-order discontinuous Galerkin scheme (2D DG2) which is more efficient and stable for realistic simulation of hydrodynamics. This modified scheme is formulated based on a local linear solution spanned by a set of local coefficients using a newly proposed cell stencil. The results show that the reformulated second-order discontinuous Galerkin scheme performs acceptably well in predicting shock propagation. The modified scheme is designed to be conservative not only for the average coefficients but also the slope coefficients, which is necessary to ensure robustness based on the well-balanced property under the lake at rest hypothesis. Our preliminary findings reveal a great potential from adopting the proposed 2D DG2 reformulation as a basis for real-world flood modelling applications.
\end{abstract}

\section{Introduction}

Godunov-type schemes have been favoured by practitioners, owing to their abilities to correctly represent non-linear conservation laws in discrete form. These formulations are basically first-order accurate in the sense that the flux balance evolves one local coefficient, which is a piecewise-constant approximation of the state variables. In the light of this philosophy, discontinuous Galerkin method is naturally an extension to high-order accuracy for a set of coefficients, that are evolved by flux balance and local data, which define a local polynomial solution. However, the number of local coefficients needed to be stored and evolved depends on the order of accuracy, spatial dimensionality and spatial resolution [1]. Consequently, the discontinuous Galerkin formulation becomes increasingly complex, costly and difficult to stabilize for real-world applications. Therefore, the second-order discontinuous Galerkin (DG2) scheme may be a selected solution as a trade-off [2].

\footnotetext{
${ }^{*}$ Corresponding author
} 
In this contribution, a well-balanced DG2 reformulation based on a simplified local cell stencil is proposed, resulting in more efficient and stable model. To demonstrate the ability of the modified DG2 formulation in realistic shallow water modelling, two tests are selected to verify that: 1) the outputs produced by this scheme acceptably matches those from the general DG2 formulation and reference solution and 2) the well-balanced property of the modified formulation is achieved, not only for the average coefficients but also, most importantly, for the slope coefficients, which is an aspect that is often overlooked when studying the well-balanced property with DG methods under the lake at rest hypothesis.

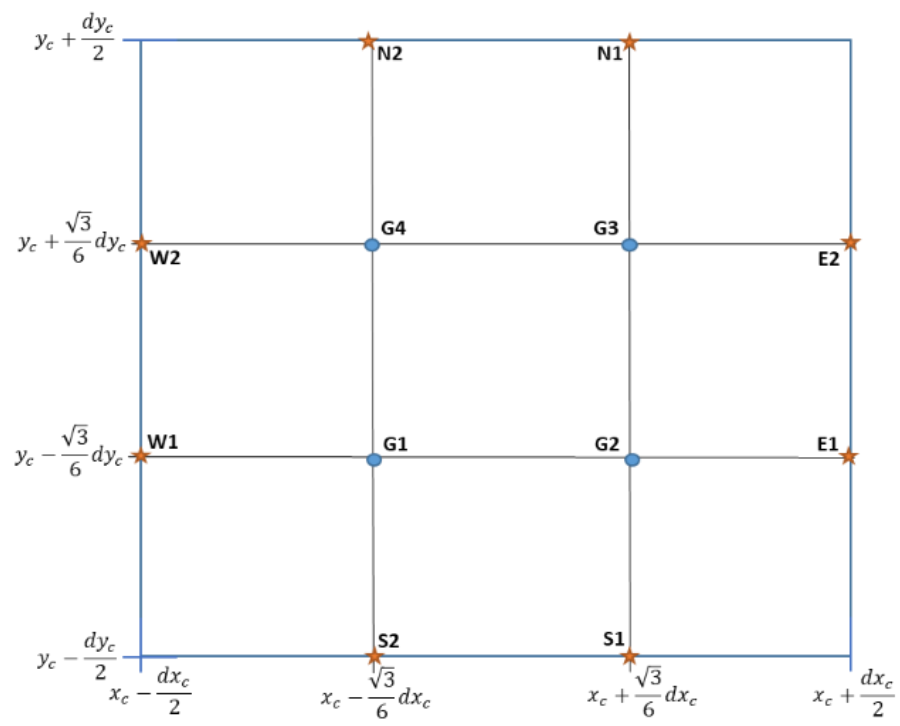

(a)

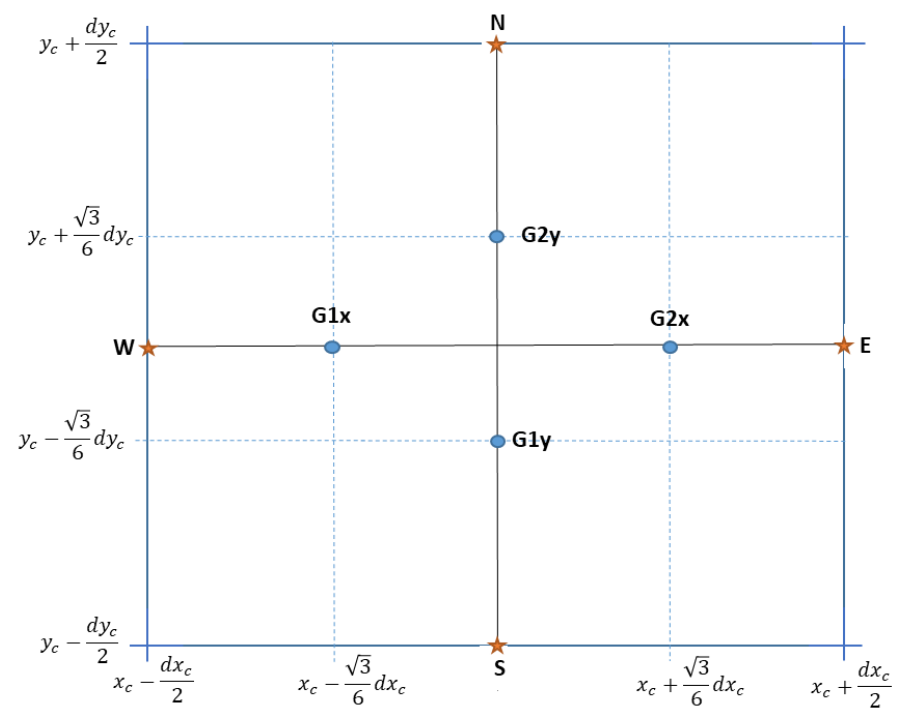

(b)

Figure 1: The local cell stencils for a) the general 2D DG2 scheme and b) the modified formulation 


\section{General 2D DG2 formulation}

The depth-averaged shallow water equation in the 2D model can be expressed as:

$$
\partial_{t} \mathbf{U}+\partial_{x} \mathbf{F}(\mathbf{U})+\partial_{y} \mathbf{G}(\mathbf{U})=\mathbf{S}(\mathbf{U})
$$

By adopting the Legendre polynomials, a solution for Eq. (1), $\mathbf{U}^{\mathbf{h}}(x, y, t)=\left[h^{h}, q_{x}^{h}, q_{y}^{h}\right]^{T}$, is sought using the local linear approximation defined by a set of coefficients, consist of the average coefficient, $\mathbf{U}_{c}^{0}$ and the slope coefficients in the $x$-direction, $\mathbf{U}_{c}^{1 x}$ and the $y$-direction, $\mathbf{U}_{c}^{1 y}$ :

$$
\left.\mathbf{U}^{\mathrm{h}}(x, y, t)\right|_{I_{c}}=\mathbf{U}_{c}^{0}(t)+\frac{\left(x-x_{c}\right)}{\left(d x_{c} / 2\right)} \mathbf{U}_{c}^{1 x}(t)+\frac{\left(y-y_{c}\right)}{\left(d y_{c} / 2\right)} \mathbf{U}_{c}^{1 y}(t)
$$

These coefficients are evolved in time by the evolution operator, $\mathbf{L}^{K}$ with $K=0,1 x$, and $1 y$, given by Eq. (3):

$$
\mathbf{L}^{K}=-\left\{\left(\mathbf{I}^{K}-\mathbf{M}^{K}\right)+\left(\mathbf{J}^{K}-\mathbf{N}^{K}\right)\right\}+\mathbf{S}^{K}
$$

where the terms $\mathbf{I}^{K}$ and $\mathbf{J}^{K}$ are the flux balance across the cell faces in the $x$ - and $y$-direction respectively, and $\mathbf{M}^{K}, \mathbf{N}^{K}$ and $\mathbf{S}^{K}$ are volume integrals relevant to local flux and source terms. This formulation specifically uses the local cell stencil shown in Figure 1(a).

The terms $\mathbf{I}^{K}$ and $\mathbf{J}^{K}$ are computed based on two evaluations of the Riemann states in the $\mathbf{x}$ - and $\mathbf{y}$ direction respectively; by this means $\mathbf{I}^{K}$ is approximated across the Eastern (E1 and E2) and Western (W1 and W2) interfaces while $\mathbf{J}^{K}$ is approximated across the Southern (S1 and S2) and Northern (N1 and N2) interfaces. $\mathbf{M}^{K}, \mathbf{N}^{K}$, and $\mathbf{S}^{K}$ are approximated via quadrature rules at four Gaussian points (G1, G2, G3 and G4). On this stencil, the general 2D DG2 formulation is computationally expensive, given by the high number of evaluations and operations needed to complete one calculation.

\section{Modified DG2 formulation for the Shallow Water Equation with bed slope term}

A reformulation is therefore proposed which is less complex, but is more efficient and stable for realistic hydraulic modelling. This scheme is formulated based on the same planar solution in Eq. (2) but using a different local cell stencil illustrated in Figure 1(b). The main benefit of this formulation is that the $x$ - and $y$-direction components are entirely decoupled. In return, the amount of evaluations and operations are reduced by half. This modified scheme also requires only one evaluation at each interface which enforce continuity for topography discretisation. 
On this stencil, Eq. (3) becomes Eq. (4a - c):

$$
\begin{aligned}
& \mathbf{L}_{c}^{0}=-\frac{1}{d x_{c}}\left(\widetilde{\mathbf{F}}_{E}-\widetilde{\mathbf{F}}_{W}\right)-\frac{1}{d y_{c}}\left(\widetilde{\mathbf{G}}_{N}-\widetilde{\mathbf{G}}_{S}\right)+\mathbf{S}\left(\mathbf{U}_{c}^{0 x}, \mathbf{U}_{c}^{0 y}, z_{c}^{1 x 1 x}, z_{c}^{1 y 1 y}\right) \\
& \mathbf{L}_{c}^{1 x}=-\frac{3}{d x_{c}}\left\{\left(\widetilde{\mathbf{F}}_{E}+\widetilde{\mathbf{F}}_{W}\right)-\left[\mathbf{F}\left(\mathbf{U}_{c}^{0 x}+\frac{\mathbf{U}_{c}^{1 x}}{\sqrt{3}}\right)-\mathbf{F}\left(\mathbf{U}_{c}^{0 x}-\frac{\mathbf{U}_{c}^{1 x}}{\sqrt{3}}\right)\right]-\frac{\sqrt{3}}{6} d x_{c}\left[\mathbf{S}\left(\mathbf{U}_{c}^{0 x}+\frac{\mathbf{U}_{c}^{1 x}}{\sqrt{3}}, z^{1 x \mid x}, z^{1 y 1 y}\right)-\mathbf{S}\left(\mathbf{U}_{c}^{0 x}-\frac{\mathbf{U}_{c}^{1 x}}{\sqrt{3}}, z^{1 x 1 x}, z^{1 y 1 y}\right)\right]\right\} \\
& \mathbf{L}_{c}^{1 y}=-\frac{3}{d y_{c}}\left\{\left(\widetilde{\mathbf{G}}_{N}+\widetilde{\mathbf{G}}_{S}\right)-\left[\mathbf{G}\left(\mathbf{U}_{c}^{0 y}+\frac{\mathbf{U}_{c}^{1 y}}{\sqrt{3}}\right)-\mathbf{G}\left(\mathbf{U}_{c}^{0 y}-\frac{\mathbf{U}_{c}^{1 y}}{\sqrt{3}}\right)\right]-\frac{\sqrt{3}}{6} d y_{c}\left[\mathbf{S}\left(\mathbf{U}_{c}^{0 y}+\frac{\mathbf{U}_{c}^{1 y}}{\sqrt{3}}, z^{1 x 1 x}, z^{1 y 1 y}\right)-\mathbf{S}\left(\mathbf{U}_{c}^{0 y}-\frac{\mathbf{U}_{c}^{1 y}}{\sqrt{3}}, z^{1 x x x}, z^{1 y 1 y}\right)\right]\right\}
\end{aligned}
$$

In Eq. (4), the wetting and drying reconstruction of the Riemann states with topography is implemented. This leads to a revised set of positivity-preserving Riemann states applied to all interfaces (proposed by [3]). For instance, at the Eastern cell interface, the states are $\left\{z_{E}^{ \pm, *}, \mathbf{U}_{E}^{-, *}, \mathbf{U}_{E}^{+* *}\right\}$. These improved states must be used to reconstruct positivity-preserving coefficients ([4], [5]) shown in Eq. (5):

Fluxes:

$$
\begin{aligned}
& \overline{\mathbf{U}}^{0 x}=\left(\mathbf{U}_{E}^{-, *}+\mathbf{U}_{W}^{+, *}\right) / 2 \text { and } \overline{\mathbf{U}}^{0 y}=\left(\mathbf{U}_{N}^{-, *}+\mathbf{U}_{S}^{+, * *}\right) / 2 \\
& \overline{\mathbf{U}}^{1 x}=\left(\mathbf{U}_{E}^{-, *}-\mathbf{U}_{W}^{+, *}\right) / 2 \quad \overline{\mathbf{U}}^{1 y}=\left(\mathbf{U}_{N}^{-, *}-\mathbf{U}_{S}^{+, *}\right) / 2 \\
& \bar{z}^{-0 x}=\left(z_{E}^{ \pm, *}+z_{W}^{ \pm, *}\right) / 2 \text { and } \bar{z}^{-0 y}=\left(z_{N}^{ \pm, *}+z_{S}^{ \pm, *}\right) / 2 \\
& \bar{z}^{-1 x 1 x}=\left(z_{E}^{ \pm, *}-z_{W}^{ \pm, *}\right) / 2 \quad \bar{z}^{-1 y 1 y}=\left(z_{N}^{ \pm, *}-z_{S}^{ \pm, *}\right) / 2
\end{aligned}
$$

With these, all components of $\mathbf{L}_{c}^{0}$ achieve the well-balanced property, but not in the case of $\mathbf{L}_{c}^{1 x}$ and $\mathbf{L}_{c}^{1 y}$. This is because the source term approximation in these operators still requires topographic information from across both the $x$ - and $y$-direction. Therefore, to regain well-balanced slope coefficients, bed slope coefficients $z^{1 y 1 y}$ and $z^{1 x 1 x}$ in Eq. (4b-4c) are replaced with $z^{-1 x y y}$ and $z^{-1 y 1 x}$ in Eq. (6) respectively, which are actually null coefficients aimed to ensure that bed slope evaluations done only in a single direction.

$$
z^{-1 x 1 y}=0 \quad \text { and } \quad \bar{z}^{-1 y 1 x}=0
$$

\section{Results and Discussions}

Coupled with the Runge-Kutta (RK) time integration method, the two 2D DG2 schemes (2DRKDG2) are compared in this section. As both schemes can generally achieve second-order accurate mesh convergence ([4], [6]), only qualitative results will be presented. Therefore, two numerical tests are selected for this purpose. The first test uses a well-known radial dam break case, which intends to verify that the modified 2D RKDG2 scheme is able to produce sufficiently accurate outputs as the general version. The second test comprises a still water state simulation over challenging scenarios involving an uneven topography with wet/dry fronts. This is done to investigate the role of the Eq. (6) in the topography discretisation to ensure that modified 2D RKDG2 scheme is able to preserve the wellbalanced property for both the average and slope discharges. 

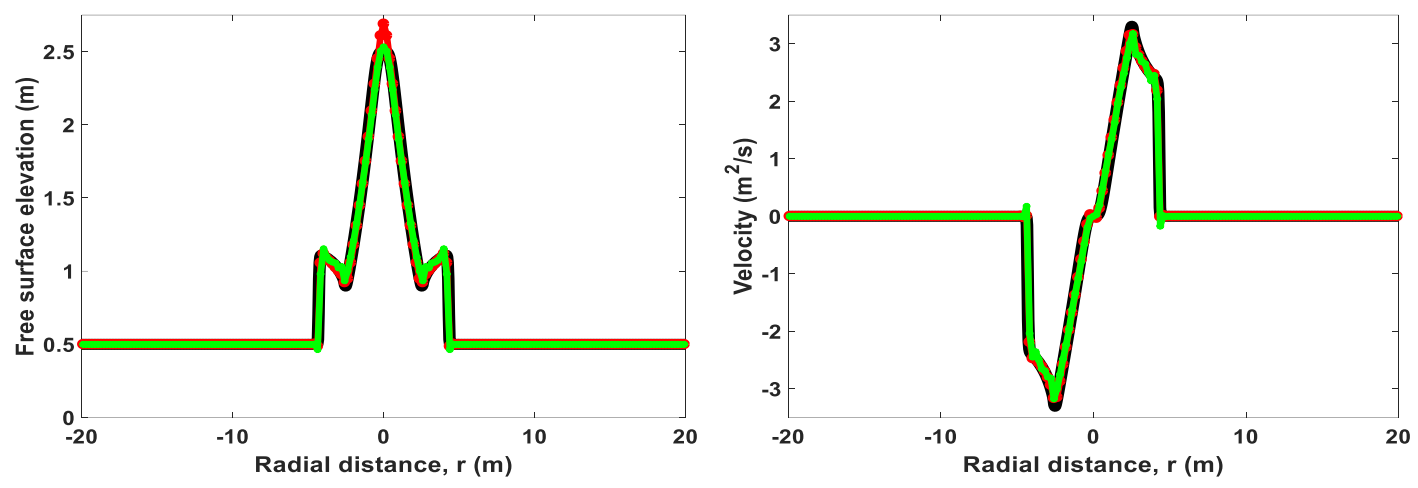

(a)
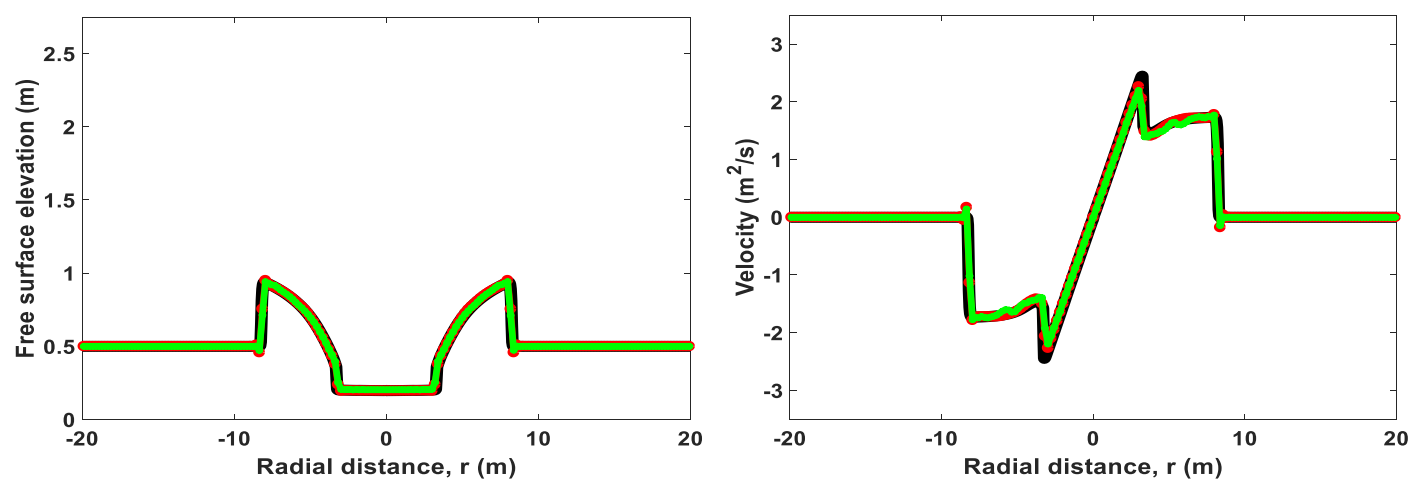

(b)
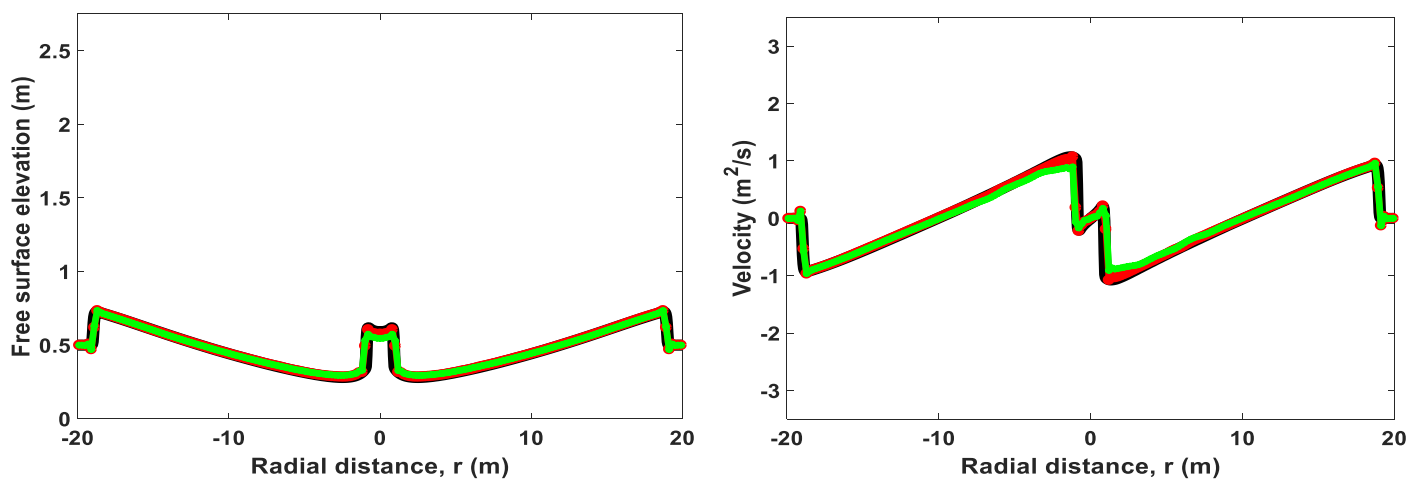

(c)

Figure 2: Free surface elevation and velocity predictions using the general (red line) and modified (green line) 2D RKDG2 schemes vs. a reference solution (black line): a) $t=0.4 \mathrm{~s}, \mathrm{~b}$ ) $t=1.4 \mathrm{~s}$ and c) $t=4.7 \mathrm{~s}$ 


\subsection{Radial dam break}

The first test was proposed in [7]. It requires a simulation of a spontaneous collapse of a radial dam over a $40 \mathrm{~m} \times 40 \mathrm{~m}$ flat and frictionless domain. The dam is centred at $x_{c}=0$ and $y_{c}=0$. The simulation is initialised with a $2.5 \mathrm{~m}$ water depth within the radial wall and $0.5 \mathrm{~m}$ on the outside of the wall. The initial velocities in the $\mathrm{x}$-direction, $u$ and the y-direction, $v$ are set to zero. Calculations on a grid with $201 \times 201$ quadrilateral cells are extracted at the radial centreline and compared with the solutions obtained by the general 2D RKDG2 scheme at $t=0.4 \mathrm{~s}, 1.4 \mathrm{~s}$ and $4.7 \mathrm{~s}$ and a $1 \mathrm{D}$ reference solution produced using a second-order MUSCL finite volume solver of the governing equations in the radial direction using 1001 cells. The slice plots of the resulting free surface elevation and velocity from both the RKDG2 schemes and reference solution are shown in Figure 2.

Immediately after the dam collapse, a primary shock wave began to propagate away from the centre of the domain. Meanwhile, a rarefaction wave began to converge inwardly and reaches the centre at $t=$ $0.4 \mathrm{~s}$ as shown in Figure 2(a). At this time, well-defined depth gradients have formed behind the primary shock wave. Once the shock wave has fully imploded in the centre, the shock wave is then reflected and began move in the opposite direction. While doing so, the free surface began to gradually drop below the initial water depth outside of the dam. This is clearly illustrated in Figure 2(b) when $t=1.4$ s. A secondary shock is also formed at this time, indicated by the formation of small, sharp velocity profile just behind the primary shock wave. When the primary shock wave is almost reaching the boundary, the secondary shock wave converges in the centre and eventually imploded at $t=4.7 \mathrm{~s}$ as illustrated in Figure 2(c). A small dip is formed in the centre of the free surface when the secondary shock wave is reflected and began to move towards the boundary.

As seen in Figure 2(a)-(c), the general 2D RKDG2 scheme is able to capture much accurately the sharp profiles of the reference solution. An exception is seen at $t=0.4 \mathrm{~s}$, where this scheme overestimated the peak free surface. This is likely due to the oversensitivity of the scheme to slope variations, contributed by the effect of cross-dimensional dependency in the flux evaluations. The modified 2D RKDG2 scheme is slightly diffusive, as evidently shown at $t=4.7 \mathrm{~s}$ when trying to produce the velocity profile of the secondary shock wave. This could possibly be contributed by the simplified hypothesis for slope-decoupling. Despite this, the modified scheme is still able to replicate the small dip at $t=4.7 \mathrm{~s}$, which is difficult to resolve numerically, but is likely to require a fine mesh to deliver the same predictive accuracy. Hence, the outcome of this test has shown that the modified 2D RKDG2 scheme can produce reasonably good results that are on par with those from the general scheme and also the reference solution.

\subsection{Still water case over uneven topography shapes}

From the previous test, it is proven that the modified 2D RKDG2 scheme can provide satisfactorily agreeable solution over a flat and frictionless topography. In this second test, the modified 2D RKDG2 scheme is further tested to assess its ability in preserving the well-balanced property over a frictionless uneven topography. This involves simulating a still water flow in a $75 \mathrm{~m} \times 30 \mathrm{~m}$ confined pool while resolving three different flow cases involving the presence of a wet/dry front (the peak of the hump is above water surface), a critically wet ( $h=0$ at the peak), and a fully wet topography (the peak is submerged underwater). The full 2D topography approximation is illustrated in Figure 3 and described in Eq. (7):

$$
z(x, y)=\max \left[0,1-\frac{1}{5} \sqrt{(x-20)^{2}+(y-15)^{2}}, 2-\frac{1}{2} \sqrt{(x-40)^{2}+(y-15)^{2}}, 3-\frac{3}{10} \sqrt{(x-60)^{2}+(y-15)^{2}}\right]
$$


The model domain is made of quadrilateral cells with mesh size of $1 \mathrm{~m}^{2}$. The initial free surface elevation is set at $h+z=1.78 \mathrm{~m}$ and the initial discharges, $q_{x}$ and $q_{y}$, are zero. All the simulations are run up to $t=100 \mathrm{~s}$.

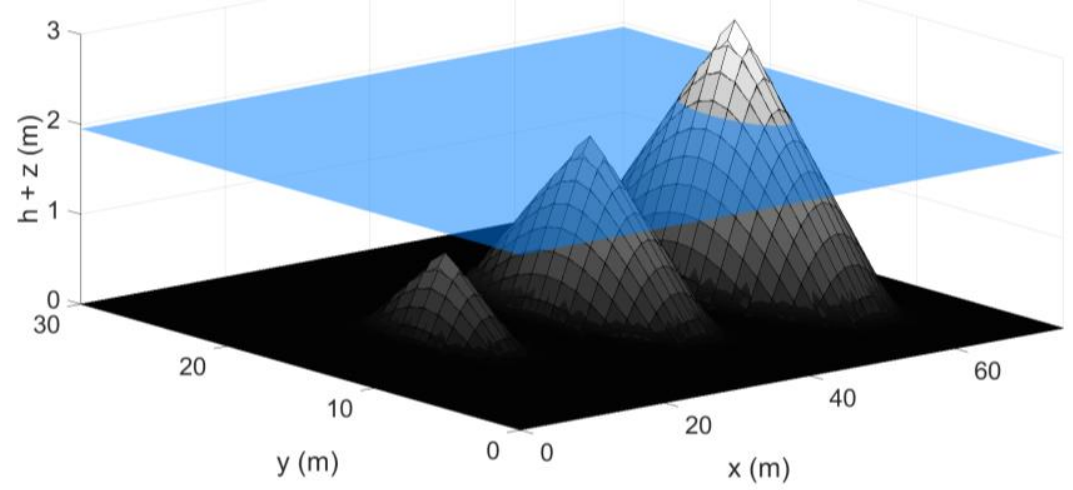

Figure 3: The initial water level and the DG2 topography approximation in the quiescent flow test

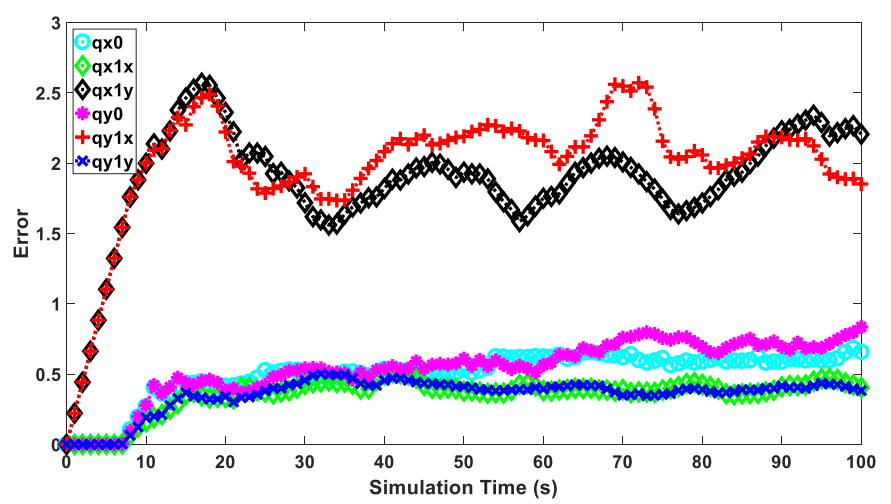

(a)

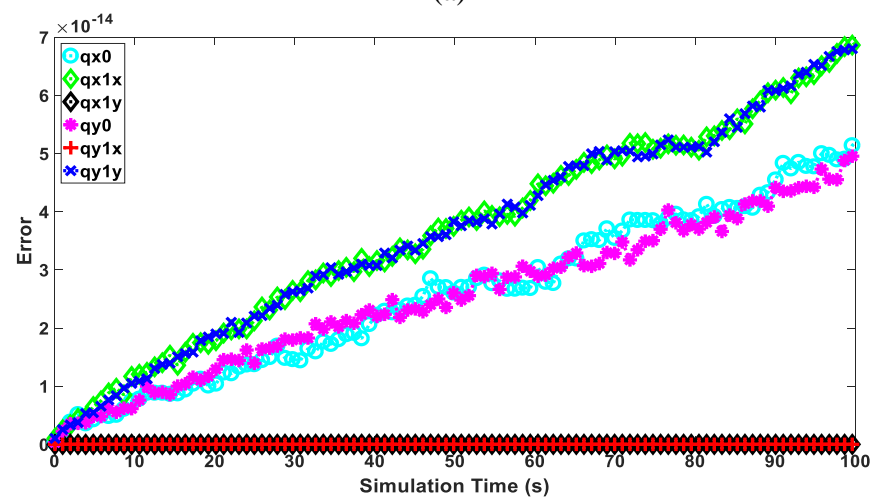

(b)

Figure 4: The errors plotted over time for the still water flow test ran a) without and b) with conditions in Eq. (6) 
First, the still water flow is simulated without imposing Eq. (6). The resulting errors for the average and slope discharges are plotted against the simulation time, illustrated in Figure 4(a). As expected, the average discharges are within the range of machine precision, indicating that these discharges are wellbalanced. The same results are seen for the slope discharges in the main direction, $q_{x}^{1 x}$ and $q_{y}^{1 y}$. However, the slope discharges in the alternate direction, i.e. $q_{x}^{1 y}$ and $q_{y}^{1 x}$, show signs of perturbations from the very start of the simulation. This is most likely triggered by the cross-dimensional slope dependency issues in the topography discretisation, as the flux evaluations in this scheme are already cross-dimensionally independent. This perturbation will eventually affect other local discharges which were initially well-balanced. At $t=100 \mathrm{~s}$, the average and all the slope discharges are slightly disturbed and hence can be said that they are not able to preserve the well-balanced state after long time evolution.

Next, the still water flow simulation is rerun, however with the activation of Eq. (6). This approach ensures that both the flux and topography discretisation are entirely cross-dimensionally independent. The resulting errors of the average and slope discharges over time is illustrated in Figure 4(b). Here, unlike the previous simulation, the errors of all the average and slope discharges are within the range of machine precision throughout the simulation. This indicates that the modified scheme is able to produce well-balanced prediction not only for the average discharges, but also for the slope discharges for all three flow cases. While there is a trend of slight increase on the errors' magnitude throughout the simulation, the rate of increase is rather small and so insignificant. Hence, the preservation of the wellbalanced property for all the discharges can be ensured for a very long time.

\section{Acknowledgement}

J. L. Ayog wish to acknowledge the support of the Ministry of Higher Education, Malaysia and Universiti Malaysia Sabah. This study is further supported by the UK Engineering and Physical Science Research Council (via grant EP/R007349/1).

\section{Reference}

[1] D. Caviedes-Voullième and G. Kesserwani, "Benchmarking a multiresolution discontinuous Galerkin shallow water model: Implications for computational hydraulics," Adv. Water Resour., vol. 86, pp. 14-31, 2015.

[2] G. Kesserwani and Y. Wang, "Discontinuous galerkin flood model formulation: Luxury or necessity?," Water Resour. Res., vol. 50, pp. 6522-6541, 2014.

[3] Q. Liang, "Flood Simulation Using a Well-Balanced Shallow Flow Model," J. Hydraul. Eng., vol. 136, no. September, pp. 669-675, 2010.

[4] G. Kesserwani and Q. Liang, "A discontinuous Galerkin algorithm for the two-dimensional shallow water equations," Comput. Methods Appl. Mech. Eng., vol. 199, no. 49-52, pp. 3356$3368,2010$.

[5] G. Kesserwani and Q. Liang, "Locally limited and fully conserved RKDG2 shallow water solutions with wetting and drying," J. Sci. Comput., vol. 50, no. 1, pp. 120-144, 2012.

[6] B. Cockburn and C.-W. Shu, "Runge - Kutta Discontinuous Galerkin Methods for ConvectionDominated Problems," J. Sci. Comput., vol. 16, no. 3, pp. 173-261, 2001.

[7] E. F. Toro, Shock-Capturing Methods for Free-Surface Shallow Flows. West Sussex: John Wiley \& Sons, 2001. 